\title{
Non-Tychonoff e-Compactifiable Spaces
}

\author{
K. P. Hart; J. Vermeer \\ Proceedings of the American Mathematical Society, Vol. 89, No. 4. (Dec., 1983), pp. 725-729.
}

Stable URL:

http://links.jstor.org/sici?sici=0002-9939\%28198312\%2989\%3A4\%3C725\%3ANS\%3E2.0.CO\%3B2-E

Proceedings of the American Mathematical Society is currently published by American Mathematical Society.

Your use of the JSTOR archive indicates your acceptance of JSTOR's Terms and Conditions of Use, available at

http://www.jstor.org/about/terms.html. JSTOR's Terms and Conditions of Use provides, in part, that unless you have obtained prior permission, you may not download an entire issue of a journal or multiple copies of articles, and you may use content in the JSTOR archive only for your personal, non-commercial use.

Please contact the publisher regarding any further use of this work. Publisher contact information may be obtained at http://www.jstor.org/journals/ams.html.

Each copy of any part of a JSTOR transmission must contain the same copyright notice that appears on the screen or printed page of such transmission.

The JSTOR Archive is a trusted digital repository providing for long-term preservation and access to leading academic journals and scholarly literature from around the world. The Archive is supported by libraries, scholarly societies, publishers, and foundations. It is an initiative of JSTOR, a not-for-profit organization with a mission to help the scholarly community take advantage of advances in technology. For more information regarding JSTOR, please contact support@jstor.org. 


\title{
NON-TYCHONOFF $e$-COMPACTIFIABLE SPACES
}

\author{
K. P. HART AND J. VERMEER
}

\begin{abstract}
We construct a non-Tychonoff space $X$ which is $e$-compactifiable, thus answering a question of S. Hechler. We also answer a question of R. M. Stephenson: whether there exists a Tychonoff space, the largest $e$-compactification of which has a noncompact semiregularization.
\end{abstract}

1. Introduction. All spaces are Hausdorff. In [He] S. Hechler introduced the class of $e$-compactifiable spaces, i.e. spaces which admit an $e$-compactification. He posed the question whether there exist non-Tychonoff $e$-compactifiable spaces. We show that such spaces exist. In [St] R. M. Stephenson observed that an $e$-compactifiable space has a largest $e$-compactification $e X$, and he asked whether the space $(e X)_{S}$ - the semiregularization of $e X$-is always compact. We show that this need not be the case, even if the space $X$ is assumed to be Tychonoff. The example of the space we present is based on an example of $\mathrm{J}$. Chaber.

\section{Preliminary definitions and theorems.}

Definition 2.1 [He]. Let $D$ be a dense subspace of $X$. $X$ is said to be e-compact with respect to $D$ if each open cover of $X$ contains a finite subcollection that covers $D$. If so, $X$ is called an e-compactification of $D$ and $D$ is called e-compactifiable.

Observe that within this terminology the expression "let $X$ be an e-compact space" is meaningless. From this definition it readily follows that an $e$-compactification of a space $X$ is an $H$-closed extension. The following theorem shows that the converse need not be true.

THEOREM $2.2[\mathrm{He}]$. Let $p X$ be an extension of $X$. Then the following statements are equivalent:

(i) $p X$ is an e-compactification of $X$.

(ii) Every ultrafilter on $X$ has an accumulation point in $p X$.

(iii) $p X$ is $H$-closed and $X \cup\{q\}$ is regular, for all $q \in p X$.

It follows that an $e$-compactifiable space is regular. The converse is not the case. From 2.2(iii) we can conclude that each noncompact $\Re$-closed space (i.e. a regular space which is closed in every regular space in which it is embedded, see [BS]) is an example of a regular non-e-compactifiable space. It is clear that every Tychonoff space is $e$-compactifiable, and in [He] the question appeared whether the converse

Received by the editors February 21, 1983.

1980 Mathematics Subject Classification. Primary 54C10, 54D20, 54G20; Secondary 54D25.

Key words and phrases. e-compactifiable spaces, perfect maps. 
holds. In the next section we show that this is not the case. We were unable to characterize the class of $e$-compactifiable spaces in terms of some separation property.

The following properties of $e$-compactifiable spaces are known.

THEOREM 2.3 [He]. (i) Let $p X$ be an e-compactification of $X$. Then $\operatorname{cl}_{p X} Y$ is an e-compactification of $Y$, for each $Y \subset X$.

(ii) Let $p_{1} X_{t}$ be an e-compactification of $X_{l}(i \in I)$. Then $\prod_{1} p_{1} X_{1}$ is an e-compactification of $\Pi X_{l}$.

Recall that a subset $U \subset X$ is regular-closed if clint $U=U$. The collection of regular-closed subsets of $X$ is a closed base for some topology on $X$. $X$ supplied with this topology is called the semiregularization of $X$, to be denoted by $X_{S}$. $X$ is called semiregular if $X$ is homeomorphic to $X_{S}$.

In [St] R. M. Stephenson observed that Theorem 2.3 implies that each $e$-compactifiable space $X$ has a largest $e$-compactification $e X$, i.e. if $\alpha X$ is an $e$-compactification of $X$ then the map id: $X \rightarrow \alpha X$ has a continuous extension over $e X$.

TheOREM 2.4. (i) [St] Let $X$ be an e-compactifiable space. Then $X$ is an open subspace of $e X$ and $e X-X$ is a closed discrete subspace of $e X$.

(ii) Let $f: X \rightarrow Y$ be a continuous map and assume that both $X$ and $Y$ are e-compactifiable. Then there is a continuous extension ef: $e X \rightarrow e Y$ of $f$.

Proof. (ii) According to 2.3(ii) we have that $e X \times e Y$ is an $e$-compactification of $X \times Y$. Define $\tilde{X}=\{(x, f(x)): x \in X\} \subset X \times Y . \tilde{X}$ is a closed subset of $X \times Y$ and $\Pi_{X} \uparrow \tilde{X}: \tilde{X} \rightarrow X$ is a homeomorphism. Since $\operatorname{cl}_{e X \times e Y} \tilde{X}$ is an $e$-compactification of $\tilde{X}$, the map $\left(\Pi_{X} \uparrow \tilde{X}\right)^{-1}: X \rightarrow \tilde{X}$ has an extension $e\left(\Pi_{X} \uparrow \tilde{X}\right)^{-1}: e X \rightarrow \operatorname{cl}_{e X \times e_{Y}} \tilde{X}$. Define ef $=\Pi_{e Y} \circ e\left(\Pi_{X} \uparrow \tilde{X}\right)^{-1}$.

As a method to answer the question of S. Hechler, R. M. Stephenson asked the following question.

"Let $X$ be an $e$-compactifiable space. Is the space $(e X)_{S}$ always compact?"

Our example of a non-Tychonoff $e$-compactifiable space provides a negative answer to this question. A partial positive answer to Stephenson's question is the following

THEOREM 2.5 [St]. Let $X$ be a regular space. If disjoint regular closed sets are contained in disjoint open subsets (in particular, if $X$ is normal), then $X$ is Tychonoff (hence e-compactifiable) and $(e X)_{S}$ is compact.

Our second example shows that the answer is negative if $X$ is only assumed to be Tychonoff. The following simple lemma is one of the keys to the construction.

Lemma 2.6. Let $X$ be a Tychonoff space. Then $(e X)_{S}$ is compact iff the map e(id): $e X \rightarrow \beta X$ is injective.

Proof. Observe that $X$ is a subspace of $(e X)_{S}$ and that the map $e(\mathrm{id}):(e X)_{S} \rightarrow \beta X$ is also continuous. Then we have " $\rightarrow$ ", since $(e X)_{S}$ is a compactification of $X$ and " $\leftarrow$ " holds because $(e X)_{S}$ is minimal Hausdorff and the topology of $\beta X$ is weaker than that of $(e X)_{S}$. 
3. The results. The following theorem is the key to our construction of a non-Tychonoff $e$-compactifiable space.

THEOREM 3.1. Perfect preimages of e-compactifiable spaces are e-compactifiable.

Proof. Let $X$ be an $e$-compactifiable space and let $f: Y \rightarrow X$ be a perfect map. We construct an $e$-compactification $\alpha Y$ of $Y$ in the following way. The underlying set of $\alpha Y$ is $Y \oplus(e X-X)$ and a topology is defined by

(i) $Y$ is open in $\alpha Y$;

(ii) For $p \in \alpha Y-Y=e X-X$ the collection $\mathscr{Q}_{p}=\left\{\{p\} \cup f^{-1}(X \cap U): U\right.$ open in $e X \& p \in U\}$ is taken as a local base in $p \in \alpha Y$.

One readily sees that $\alpha Y$ is a Hausdorff extension of $Y$. To see that $\alpha Y$ is an $e$-compactification of $Y$, consider an ultrafilter $\mathscr{F}$ on $Y$. Then $f(\mathscr{F})=\{f(F): F \in \mathscr{F}\}$ is an ultrafilter on $X$; hence $f(\mathscr{F})$ has an accumulation point $q$ in $e X$. If $q \in X$ then, since $f$ is perfect, $\mathscr{F}$ has an accumulation point in $f^{-1}(q)$. If $q \in e X-X$, then $f(F) \cap U_{q} \neq \varnothing$ for each open neighborhood $U_{q}$ of $q$ in $e X$ and $F \in \mathscr{F}$. Since $f(F) \subset X$ it follows that $F \cap f^{-1}\left(U_{q} \cap X\right) \neq \varnothing$, i.e. $q$-considered as an element of $\alpha Y$-is an accumulation point of $\mathscr{F}$.

In [Ch] J. Chaber constructed examples of non-Tychonoff perfect preimages of Tychonoff spaces, and so these examples establish the existence of non-Tychonoff $e$-compactifiable spaces. From 2.3(i) it follows that subspaces of perfect preimages of Tychonoff spaces are $e$-compactifiable. We were not able to construct $e$-compactifiable spaces outside this particular class. Observe that a space $X$ in this class (with $|X|>1)$ admits nonconstant real-valued continuous functions.

Question 3.2. Do there exist $e$-compactifiable spaces on which every real-valued continuous function is constant?

Let us now answer the question of R. M. Stephenson, whether there exist Tychonoff spaces $X$ for which $(e X)_{S}$ is not compact. Our strategy is as follows. We construct a Tychonoff space $X$, a point $p \in \beta X-X$ and an extension $\alpha X$ of $X$ such that $|\alpha X-X|>1$ and such that the map $f: \alpha X \rightarrow X \cup\{p\}(\subset \beta X)$ defined by $f(x)=x(x \in X)$ and $f(\alpha X-X)=p$ is perfect. It then follows that $\alpha X$ is $e$-compactifiable, and since $e \alpha X$ can be considered as an $e$-compactification of $X$, we can conclude from the diagram below that the map $e(\mathrm{id}): e X \rightarrow \beta X$ is not injective. ( $e_{1}$ is the extension of id: $X \rightarrow \alpha X \subset e \alpha X$ to $e X$ (see 2.4(iii)).) ( $e_{2}$ is the extension of id: $X \cup\{p\} \rightarrow \beta(X \cup\{p\})$ to $e(X \cup\{p\}$.) Indeed, the diagram shows that $e(\mathrm{id})=$ $e_{2} \circ$ ef $\circ e_{1}$; hence $e(\mathrm{id})^{-1}(p)>1$.

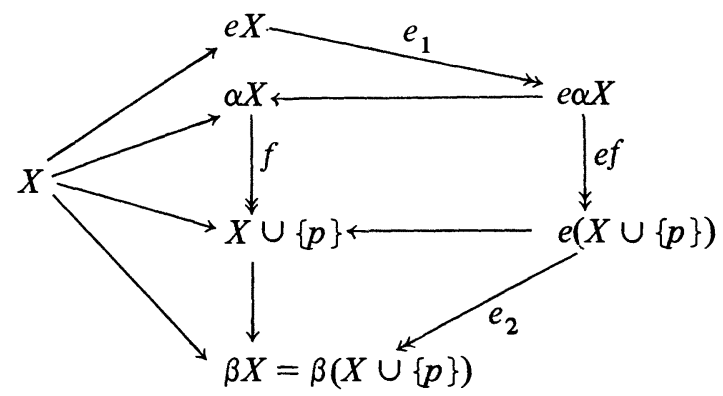


The example we present is almost identical to the one constructed by J. Chaber. The only difference lies in the fact that we want the point $p$ to lie in the Čech-Stone remainder of $X$. For the reader's convenience we give the construction in detail.

EXAMPLE 3.3. Put $T=\left(\omega_{1}+1\right) \times\left(\omega_{1}+1\right)-\left\{\left(\omega_{1}, \omega_{1}\right)\right\}$. The set of pairs of the form $\left(\alpha, \omega_{1}\right) \in T$ will be called the left edge of $T$. The set of pairs of the form $\left(\omega_{1}, \alpha\right) \in T$ will be called the right edge of $T$. Define the space $T^{n}$, for $n \in \mathbf{N}$, as the space obtained by identification in the sum $\bigoplus_{i=1}^{n} T(i)$ where $T(i)=T \times\{i\}$, of the right edge of $T(i)$ with the left edge of $T(i+1)$. Let $\varphi_{n}: \bigoplus_{i=1}^{n} T(i) \rightarrow T^{n}$ be the corresponding identification-map. For each $0 \leqslant k \leqslant n$ we define an open subset $U_{h}^{n} \subset T^{n}$, by

$$
U_{k}^{n}= \begin{cases}\text { int } \varphi_{n}(T(1)) & (k=0), \\ \text { int } \varphi_{n}(T(k) \cup T(k+1)) & (k=1, \ldots, n-1), \\ \text { int } \varphi_{n}(T(n)) & (k=n) .\end{cases}
$$

Finally we define $X=\bigoplus_{n=1}^{\infty} T^{n}$.

It is well known that $\left|\beta T^{n}-T^{n}\right|=1$, for each $n \in \mathbf{N}$. For $\alpha<\omega_{1}$ put $Z_{\alpha}=$ $\left[\alpha, \omega_{1}\right] \times\left[\alpha, \omega_{1}\right]-\left\{\left(\omega_{1}, \omega_{1}\right)\right\}$. Then $\left\{Z_{\alpha}: \alpha<\omega_{1}\right\}$ is a base for the unique nonfixed $z$-ultrafilter on $T$. If we define, for $n \in \mathbf{N}, Z_{\alpha}^{n}=\varphi_{n}\left(\oplus_{i=1}^{n}\left(Z_{\alpha} \times(i)\right)\right)$ then $\left\{Z_{\alpha}^{n}\right.$ : $\left.\alpha<\omega_{1}\right\}$ is a base for the unique nonfixed $z$-ultrafilter $\mathbf{Z}^{n}$ on $T^{n}$.

Next we define a point $p \in \beta X-X$. Let $\mathcal{G}$ be a nonfixed ultrafilter on $\mathbf{N}$. For $G \in \mathcal{G}$ and $\alpha<\omega_{1}$ put $Z(G, \alpha)=\cup\left\{Z_{\alpha}^{n}: n \in G\right\}$. It is easy to verify that the collection $\left\{Z(G, \alpha): G \in \mathcal{G}, \alpha<\omega_{1}\right\}$ is a base for a nonfixed $z$-ultrafilter $\mathscr{T}$ on $X$. Let $p \in \beta X-X$ be the point in $\beta X$ corresponding to $\mathscr{F}$, i.e. $\{p\}=\cap\left\{\operatorname{cl}_{\beta X} F: F \in \widetilde{F}\right\}$. In the space $X \cup\{p\}$ we have the following: If $U$ is open in $X$ then $U \cup\{p\}$ is a neighborhood of $p$ in $X \cup\{p\}$ iff $\exists G \in \mathcal{G} \exists \alpha<\omega_{1}$ such that $Z(G, \alpha) \subset U$. (*)

(This is not completely trivial, since $X$ is not normal. However, it follows easily by considering the space $\tilde{X}=\bigoplus_{n=1}^{\infty} \mathrm{cl}_{\beta X} T^{n} \subset \beta X$, which is $\sigma$-compact (hence normal). We omit the details.)

Let us now introduce a topology on the set $X \cup[0,1]([0,1]$ is the unit interval) in the following way. For $t \in[0,1]$ let $\left\{V_{l}(t)\right\}_{l=1}^{\infty}$ be a countable local base at $t$. For $t \in[0,1], l \in \mathbf{N}, G \in \mathcal{G}, \alpha<\omega_{1}$ define

$$
U(t, l, G, \alpha)=\bigcup_{n \in G} \bigcup_{s \in V_{l}(t)}\left(U_{[n . s]}^{n} \cap Z_{\alpha}^{n}\right) \cup V_{l}(t)
$$

(Here $[n . s]$ denctes the greatest integer not greater than n.s.) And next we put:

$X$ is open in $X \cup[0,1]$.

For $t \in[0,1]$ the collection $\left\{U(t, l, G, \alpha): l \in \mathbf{N}, G \in \mathcal{G}, \alpha<\omega_{1}\right\}$ is defined to be a local base of $t$ in $X \cup[0,1]$.

Observe that $[0,1]$ is embedded in $X \cup[0,1]$. It is easy to check that $X \cup[0,1]$ is a Hausdorff space. In fact our topology has more open sets than Chaber's.

Claim. Let $U$ be a subset of $X \cup[0,1]$ which contains [0,1]. Then $U$ is neighborhood of $[0,1]$ in $X \cup[0,1]$ iff $\exists G \in \mathcal{G} \exists \alpha<\omega_{1}$ such that $Z(G, \alpha) \subset U$. 
Proof. Assume $Z(G, \alpha) \subset U$. Then, for $t \in[0,1], t \in U(t, l, G, \alpha) \subset Z(G, \alpha)$. Hence $[0,1] \subset$ int $U$. On the other hand, assume $[0,1] \subset$ int $U$. Then, $\forall t \in[0,1]$ $\exists l(\mathrm{t}) \in \mathbf{N} \exists G(t) \in \mathcal{G} \exists \alpha(t)<\omega_{1}$ such that

$$
t \in U(t, l(t), G(t), \alpha(t)) \subset U .
$$

Since $[0,1]$ is compact, $[0,1]$ can be covered by finitely many of these sets. Say $[0,1] \subset \cup_{l=1}^{k} U\left(t_{1}, l\left(t_{1}\right), G\left(t_{1}\right), \alpha\left(t_{1}\right)\right)(\subset U)$. Put $G=\cap_{t=1}^{k} G\left(t_{l}\right)(\in \mathcal{G})$ and $\alpha=$ $\sup \left\{\alpha\left(t_{t}\right): i \leqslant k\right\} \quad\left(<\omega_{1}\right)$. We claim that $Z(G, \alpha) \subset \cup_{l=1}^{k} U\left(t_{t}, l\left(t_{t}\right), G\left(t_{t}\right), \alpha\left(t_{t}\right)\right)$ $(\subset U)$. Choose $p \in Z(G, \alpha)$, say $p \in Z_{\alpha}^{n}$ for some $n \in G$. Since $T^{n}=\cup_{k=0}^{n} U_{k}^{n}$, there exists $k \leqslant n$ such that $p \in U_{k}^{n}$. Choose $s \in[0,1]$ such that $[n . s]=k$. If $s \in U\left(t_{l}, l\left(t_{l}\right), G\left(t_{l}\right), \alpha\left(t_{l}\right)\right)$ then, since $G \subset G\left(t_{l}\right)$ and $Z_{\alpha} \subset Z_{\alpha\left(t_{l}\right)}$, we conclude that $p \in Z\left(\alpha\left(t_{1}\right)\right) \cap U_{[n . s]}^{n}$ for "some" $n \in G\left(t_{1}\right)$, i.e. $p \in U\left(t_{1}, l\left(t_{1}\right), G\left(t_{l}\right), \alpha\left(t_{1}\right)\right)$. The claim follows.

From the claim and from $(*)$ we conclude that the space obtained from $X \cup[0,1]$ by identifying $[0,1]$ to a point is homeomorphic to $X \cup\{p\}$. Obviously the map $f$ : $X \cup[0,1] \rightarrow X \cup\{p\}$ defined by $f(x)=x(x \in X)$ and $f[0,1]=p$ is a perfect map. Hence, all the required properties are satisfied.

REMARK 3.4. It is well known that each space $T^{n}$, as defined in 3.3, has a unique (nontrivial) regular extension, namely $\beta T^{n}$. It follows that $\mathrm{cl}_{e X} T^{n} \simeq \beta T^{n}$, for all $n \in \mathbf{N}$. Consider the space $\tilde{X}=\bigoplus_{n=1}^{\infty} \beta T^{n}$. Then $X \subset \tilde{X} \subset e X . \tilde{X}$ is a $\sigma$-compact, hence normal, and according to 2.5 this implies that $(e \tilde{X})_{S}=\beta \tilde{X}=\beta X$. Since $(e X)_{S} \neq \beta X$, we conclude that the map id: $\tilde{X} \rightarrow e X$ cannot be extended continuously to $e \tilde{X}$. At first glance this may seem a contradiction, but it is not. One cannot use 2.4(ii) to ensure that such an extension should exist since $e X$ is not $e$-compactifiable ( $e X$ is not even semiregular), nor the fact that $e \tilde{X}$ is the largest $e$-compactification, since $e X$ is not an $e$-compactification of $\tilde{X}$. (A nonfixed ultrafilter on $\tilde{X}-X$ does not have an accumulation point in $e X$.)

\section{REFERENCES}

[BS] M. P. Berri and R. H. Sorgenfrey, Minimal regular spaces, Proc. Amer. Math. Soc. 14 (1963), 454-458.

[Ch] J. Chaber, Remarks on open-closed mappings, Fund. Math. 73 (1971), 197-208.

[He] S. H. Hechler, On a notion of weak compactness in non-regular spaces, Studies in Topology (N. M. Stavrakas and K. R. Allen, eds.), Academic Press, New York, 1975, pp. 215-237.

[St] R. M. Stephenson, Not every minimal Hausdorff space is e-compact, Proc. Amer. Math. Soc. 52 (1975), 381-388.

Subfaculteit Wiskunde en Informatica, Vrije Universiteit, De BoelelaAn 1081, 1081 HV AmSTERdam, The NeTherlands (Current address of K. P. Hart)

Current address (J. Vermeer): Department of Mathematics, University of Kansas, Lawrence, Kansas 66045 
http://www.jstor.org

\title{
LINKED CITATIONS \\ - Page 1 of 1 -
}

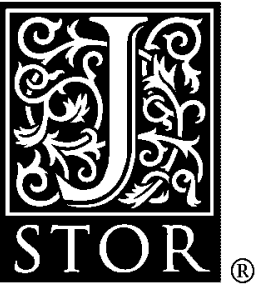

You have printed the following article:

\section{Non-Tychonoff e-Compactifiable Spaces}

K. P. Hart; J. Vermeer

Proceedings of the American Mathematical Society, Vol. 89, No. 4. (Dec., 1983), pp. 725-729.

Stable URL:

http://links.jstor.org/sici?sici=0002-9939\%28198312\%2989\%3A4\%3C725\%3ANS\%3E2.0.CO\%3B2-E

This article references the following linked citations. If you are trying to access articles from an off-campus location, you may be required to first logon via your library web site to access JSTOR. Please visit your library's website or contact a librarian to learn about options for remote access to JSTOR.

\section{References}

\author{
${ }^{\mathrm{BS}}$ Minimal Regular Spaces \\ Manuel P. Berri; R. H. Sorgenfrey \\ Proceedings of the American Mathematical Society, Vol. 14, No. 3. (Jun., 1963), pp. 454-458. \\ Stable URL: \\ http://links.jstor.org/sici?sici=0002-9939\%28196306\%2914\%3A3\%3C454\%3AMRS\%3E2.0.CO\%3B2-N

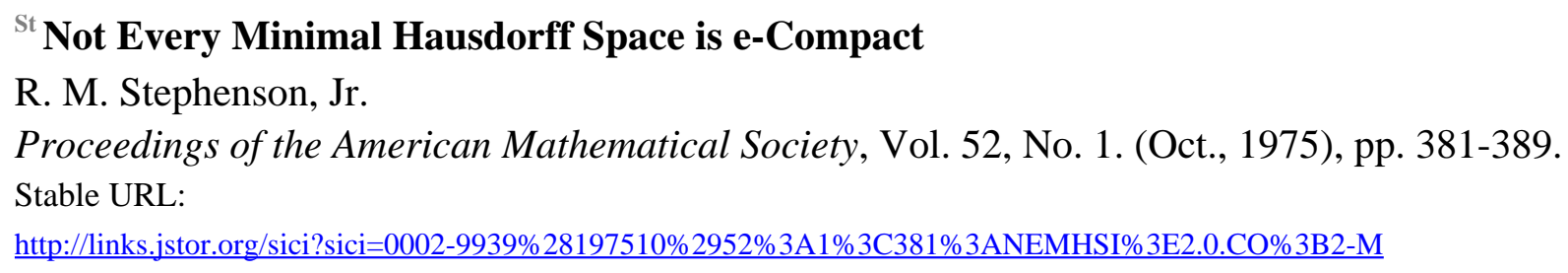

NOTE: The reference numbering from the original has been maintained in this citation list. 